\title{
Investigação de sistema epitermal por espectroscopia de refletância, imagens HyMap e magnetometria: um estudo de caso no sul do Peru
}

Thais Andressa Carrino, Departamento de Geologia/Universidade Federal de Pernambuco; Alvaro Penteado Crósta, Instituto de Geociências/Universidade Estadual de Campinas; Adalene Moreira Silva, Instituto de Geociências/Universidade de Brasília; Catarina Labouré Bemfica Toledo, Instituto de Geociências/Universidade de Brasília

Copyright 2016, SBGf - Sociedade Brasileira de Geofísica

Este texto foi preparado para a apresentação no VII Simpósio Brasileiro de Geofisica, Ouro Preto, 25 a 27 de outubro de 2016. Seu conteúdo foi revisado pelo Comitê Técnico do VII SimBGf, mas não necessariamente representa a opinião da SBGf ou de seus associados. É proibida a reprodução total ou parcial deste material para propósitos comerciais sem prévia autorização da SBG.

\section{Resumo}

Uma revisão da aplicação de métodos integrados de sensoriamento remoto hiperespectral e magnetometria, além de análises geoquímicas semiquantitativas e petrografia é apresentada num estudo de caso de caracterização de prospecto de ouro do sul do Peru. A mineralogia da alteração hidrotermal identificada, composta por fases hipógenas e supérgenas, revela um sistema epitermal de alta sulfetação. A ampla exposição em superfície da alteração é retratada por diversos núcleos de alteração, majoritariamente compostos de quartzo, minerais do grupo da alunita (Na-alunita, Kalunita, alunita intermediária, \pm Ca-alunita), dickita, caulinita ( \pm topázio, diásporo, pirofilita), apresentando similaridades com alvos adjacentes, dispostos num trend NE-SW. Zonas desmagnetizadas, por sua vez, mostram compatibilidade com a direção principal dos arcos magmáticos (NW-SE), e com a distribuição da mineralogia de alteração passível de cartografia via imagens do sensor HyMap.

\section{Introdução}

Depósitos epitermais de ouro do Mioceno compreendem as principais fontes deste metal no Peru (Acosta et al., 2008). Especificamente, o setor sul é caracterizado por depósitos de pequeno a médio porte (Figura 1), com destaque aos epitermais de alta sulfetação (Tucari, Santa Rosa) e de intermediária sulfetação (Canahuire) (Loayza et al., 2004, Santos et al., 2011).

As principais rochas hospedeiras de mineralização aurífera nesse setor compreendem rochas vulcânicas do Grupo Barroso ( 8-7 Ma: andesito, traquiandesito, brecha vulcânica e tufo de composição predominantemente andesítica a traquiandesítica), e rochas do Grupo Maure e Formação Capillune (sedimentares de origem lacustre e.g., arenito, siltito, argilito e calcário -, intercaladas com tufo de composição traquiandesítica, dacítica e riolítica; 11-8 Ma) (Palácios, 1995, Benavides-Cáceres, 1999), e, ocasionalmente, no caso peculiar de Canahuire, rochas carbonáticas juro-cretáceas (Grupo Yura), e brechas freáticas e freatomagmáticas (Santos et al. 2011).

A boa exposição de afloramentos nessa região do Peru torna a aquisição e a análise sistemática de dados de espectroscopia de refletância e de imageamento extremamente favoráveis à investigação de alvos exploratórios, sobretudo quando integrados com dados complementares, caso de dados magnetométricos, petrográficos e geoquímicos (e.g., EDS-MEV, DRX).

Nesse contexto, este artigo apresenta uma investigação de dados multifonte direcionada à caracterização do prospecto Chapi Chiara (Fig. 1), baseado na compilação de trabalhos previamente produzidos pelos autores (Carrino et al., 2015a, b; 2016 - submetido), com foco na exemplificação do uso de geotecnologias como ferramenta potencialmente útil à atividade de exploração mineral.

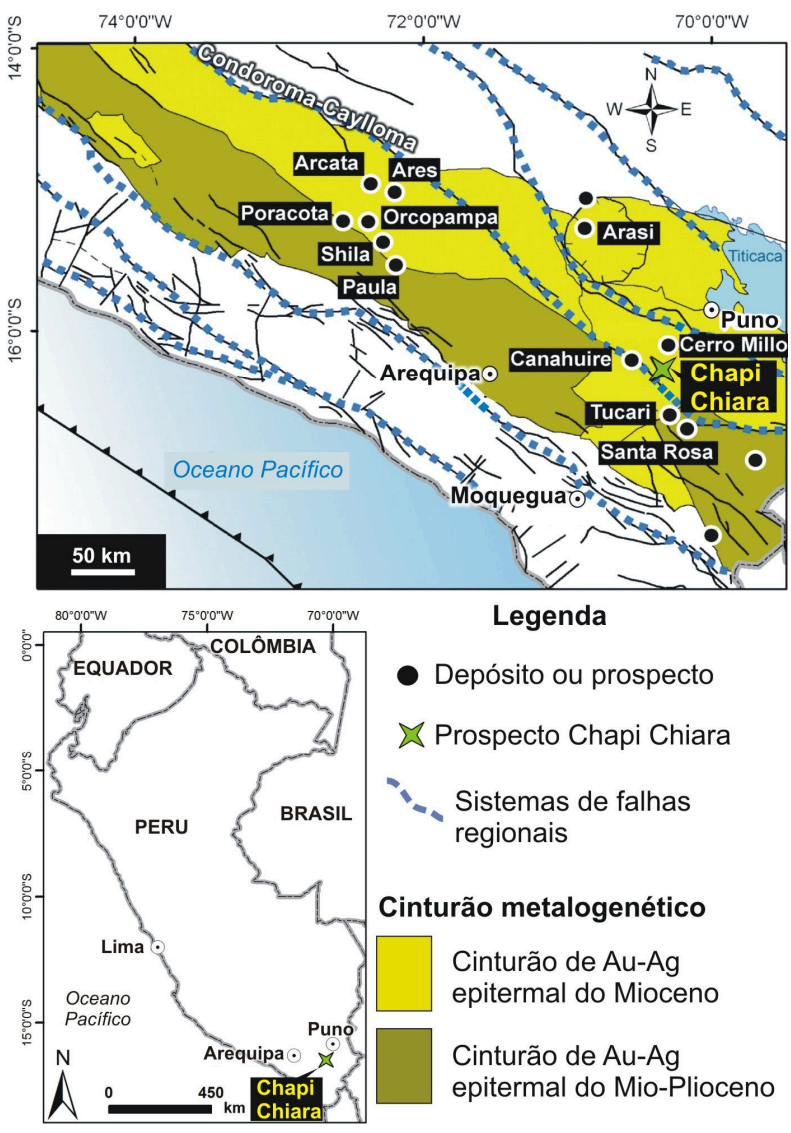

Figura 1. Localização do prospecto Chapi Chiara no sul do Peru (modificado de Acosta et al., 2008).

\section{Materiais}

O conjunto de dados multifonte disponíveis ao estudo compreende: 
(1) 671 curvas espectrais obtidas de amostras de rochas via o emprego do espectrorradiômetro TerraSpec ${ }^{\mathrm{TM}}$ (Analytical Spectral Devices), caracterizado por mais de 2000 canais espectrais;

(2) oito faixas de voo do sensor hiperespectral HyMap (Hyperspectral Mapper) coletadas em julho de 2010. Estes dados são caracterizados por 125 bandas espectrais, e por resolução espacial de $5 \mathrm{~m}$. As imagens foram cedidas pela empresa HyVista Corporation já previamente processadas quanto às correções geométricas e atmosféricas;

(3) medidas de susceptibilidade magnética associadas a 16 amostras de rochas. Estas medidas foram obtidas por meio do uso do equipamento Hand-held conductivity \& magnetic susceptibility meter GDD (Instrumentation GDD, Inc.), portador de uma sonda manual;

(4) dados magnetométricos terrestres adquiridos pela empresa Zissou Peru em agosto de 2011. Os dados foram coletados mediante uso de três magnetômetros POS Systems Newmont, e totalizam 58 linhas de aquisição N-S, com medidas espaçadas entre si variando entre 1 e $5 \mathrm{~m}$, aproximadamente. As medidas $\mathrm{E}-\mathrm{W}$ foram espaçadas em torno de $50 \mathrm{~m}$;

(5) 37 lâminas delgadas de amostras de rochas;

(6) microanálises químicas semiquantitativas adquiridas com microscópios eletrônicos de varredura (MEV) Zeiss LEO 430i e Zeiss EVO MA15, acoplados a espectrômetros de energia dispersiva de raios-X (EDS);

(7) dados de difratometria de raios-X (DRX - método do pó) obtidos com o difratômetro de raios-X Ultima IV da Rigaku, equipado com tubo de cobre.

\section{Métodos}

As abordagens metodológicas adotadas neste estudo se resumem a duas etapas de investigação: (1) etapa de investigação de fases minerais (alteração), e (2) etapa de análise e interpretação dos padrões de espacialização das áreas alteradas.

Com relação à primeira etapa, as fases minerais de alteração foram identificadas a partir da integração de análises via petrografia ótica, MEV-EDS, DRX e interpretação visual das feições de absorção de curvas espectrais obtidas com o TerraSpec ${ }^{\mathrm{TM}}$. Com base no discernimento das fases minerais hipógenas e supérgenas, visou-se a identificação dos principais tipos de alteração hidrotermal e, por conseguinte, com base nas evidências mineralógicas e texturais, do tipo de sistema mineralizador.

Em função das boas condições de exposição de afloramentos na região, foi possível realizar a etapa de análise e interpretação da espacialização das áreas alteradas. Neste contexto, foram selecionadas curvas espectrais de minerais de alteração como endmembers para o processamento das imagens do sensor HyMap, mediante o uso da técnica de classificação supervisionada MTMF (Mixture Tunned Matched Filtering;
Boardman et al., 1995, Mundt et al., 2007). A partir desta metodologia, foi possível gerar um mapa da alteração presente na área do prospecto Chapi Chiara, com a finalidade de se identificar e interpretar diferentes núcleos de alteração e suas relações espaciais com trends estruturais.

Complementarmente, foram investigadas as respostas de susceptibilidade magnética de amostras de rochas, e foi feita a interpretação de padrões de desmagnetização em produtos magnetométricos, caso da imagem da amplitude do sinal analítico (ASA - Nabighian, 1972, 1974, Roest et al., 1992). Estas respostas foram associadas às informações mineralógicas e texturais obtidas por meio das análises dos demais dados multifonte acima citados.

\section{Resultados e discussão}

A região do prospecto Chapi Chiara é caracterizada pela presença de andesito e vulcânicas ácidas, além de rochas hidrotermalizadas, representativas de três tipos principais de zoneamento: argílica avançada, argílica e propilítica.

A resposta magnética de rochas vulcânicas é caracterizada por valores de susceptibilidade magnética da ordem de $28 \times 10^{-3} \mathrm{SI}$ para andesitos, e tendem para nulos no caso de tufos riolíticos. Os valores medidos de susceptibilidade magnética são explicados pela presença de minerais ferrimagnéticos em quantidade traço, caso de titanomagnetita e magnetita (Figura 2).

A alteração argílica avançada é, espacialmente, a mais expressiva, enquanto as zonas de alteração argílica e propilítica são restritas (métricas-decamétricas).

A alteração argílica avançada é pervasiva e é caracterizada por rochas ricas em quartzo hidrotermal ( 65-90 vol.\%), com aproximadamente 10 a $30 \%$ de minerais do grupo da alunita (K-alunita, Na-alunita, $\pm \mathrm{Ca}$ alunita), por vezes contento caulinita e/ou dickita ( 3 a $\sim 20$ vol.\%). Topázio aparece como mineral acessório em muitas amostras ( 1-5 vol.\%), assim como ocorrências mais restritas de pirofilita, diásporo e pirita $(<1$ vol. \%), além de barita ( 1 vol. \%). Complementarmente, aparece rutilo como a fase de óxido mais comum, conforme verificado por petrografia e por DRX (e.g., Figura 2), totalizando $<1$ vol. $\%$. Os cristais de rutilo ocorrem de forma disseminada e são coexistentes com caulinita, alunita e quartzo. Em geral, são cristais anaedrais, de granulometria fina $(\sim 2$ a $\sim 16 \mu \mathrm{m})$, podendo, algumas vezes, apresentar granulometria da ordem de $40 \mu \mathrm{m}$.

Texturalmente, os cristais de alunita são tabulares, euedrais a subeudrais, e possuem maior tamanho ( $\sim 0 \mathrm{a}$ $150 \mu \mathrm{m}$ ) quando coexistente com topázio (e.g., ponto CC56 - Figura 2).

A alteração argílica é constituída por quartzo hidrotermal (>70 vol.\%), ilita ou ilita paragonítica ( \pm íon amônio) ( 20 vol.\%) e, em algumas amostras, aparece uma quantidade apreciável de pirita ( 1 até $\sim 7$ vol.\%) (e.g., Figura 2). Pouca esmectita pode estar associada a esta 
paragênese (1 - 5 vol.\%), além de barita ( 1 vol.\%). Rutilo compreende a principal fase de óxidos reconhecida (<1 vol.\% - cf., Figura 2), e é caracterizado por cristais anaedrais encontrados em setores ricos em pirita. São marcados por granulometria fina, da ordem de $\sim 5$ a $\sim 15$ $\mu \mathrm{m}$. Baixas respostas de susceptibilidade magnética são observadas, assim como ocorre na região da zona de alteração argílica avançada (faixa de cerca de 0 a $0,15 \times$ $10^{-3} \mathrm{SI}$ ). Isto se deve à presença de fases de $\mathrm{Fe}-\mathrm{Ti}$ paramagnéticas e antiferromagnéticas, caso do rutilo e ilmenita, respectivamente, em quantidades traço (c.f., Figura 2).

A alteração propilítica é marcada pela ocorrência de clorita e epidoto $(\sim 10$ a $\sim 20$ vol.\%), e carbonato (principalmente calcita - 5-15 vol. \%). Há quantidades subordinadas de argilominerais ( $\%$ vol. $\%)$, e sulfetos como pirita e calcopirita ( 1-3 vol.\%). É possível observar uma grande quantidade de minerais ígneos pouco alterados, caso de plagioclásio (principalmente andesina - e.g., Figura 2), além de zircão, monazita e apatita ( 1 vol.\%), e de óxidos de Fe-Ti ( 1 a $\sim 3$ vol.\%). Cristais anaedrais e subeuedrais de rutilo, ilmenita, magnetita e titanomagnetita coexistem com cristais de clorita e carbonatos (produtos de alteração de piroxênio). Rutilo aparece como lamelas em cristais de titanomagnetita ou como cristais isolados, com tamanhos variando entre $\sim 6$ e $\sim 50 \mu \mathrm{m}$. Cristais de ilmenita são caracterizados por tamanhos entre 5 e $60 \mu \mathrm{m}$, enquanto cristais de titanomagnetita e magnetita podem alcançar tamanhos de $\sim 3,6$ a $>170 \mu \mathrm{m}$. Em função da manutenção de minerais traço ferrimagnéticos de origem parental, é que se justificam as respostas similares de susceptibilidade magnética presentes em rochas da zona propilítica em relação aos andesitos aflorantes (c.f., gráfico da Figura 2 - medidas de susceptibilidade magnética na faixa de $\sim 3,45 \times 10^{-3}$ a $\sim 36,03 \times 10^{-3} \mathrm{SI}$ ). Espectralmente, a clorita domina as respostas de refletância nas amostras afetadas pela alteração do tipo propilítica (c.f., Figura 3).

A mineralogia descrita brevemente acima abrange as principais fases hipógenas reconhecidas na área do prospecto. Como fases supérgenas, foram identificadas, principalmente, a goethita e a jarosita.

O reconhecimento mineralógico é essencial à interpretação dos resultados obtidos via processamento de imagens hiperespectrais (caso do HyMap neste estudo), e de repostas magnéticas advindas de níveis crustais rasos, mediante aplicação de filtros para realce de alta frequência dos dados do campo magnético residual, caso da amplitude do sinal analítico derivadas vertical, tilt, entre outros. Desta forma, observando-se a disposição espacial das zonas de alteração hidrotermal mostradas na Figura $4 \mathrm{~A}$ com as zonas de baixas respostas de amplitude do sinal analítico na Figura 4B, pode-se analisar e interpretar:

(1) Extensos núcleos de alteração argílica avançada, com domínio de minerais do grupo da alunita e minerais do grupo da caulinita, são observados nos setores sudoeste e nordeste da região, conforme observado na Figura 4A. Especificamente, é constatada a exposição de minerais hipógenos formados em condições de maior temperatura (acima de $\sim 200-250^{\circ} \mathrm{C}$ - White e Hedenquist, 1995, Hedenquist et al., 2000), caso de alunita + topázio, alunita + dickita + pirofilita, diásporo, e alunita de composição intermediária.

(2) Rochas intensamente alteradas (alterações argílica avançada e argílica), caracterizadas por minerais paramagnéticos, diamagnéticos e antiferromagnéticos, estão associadas com respostas de baixa amplitude do sinal analítico (Figura 4B). Rochas vulcânicas e aquelas afetas por alteração propilítica estão relacionadas com maiores valores da amplitude do sinal analítico.

(3) As estruturas NW-SE de desmagnetização observadas na imagem da amplitude do sinal analítico (Figura 4B) são compatíveis com o principal condicionamento estrutural regional, representado pelo sistema de falhas Condoroma-Caylloma (c.f., Figura 1), sendo paralelas à orientação de arcos vulcânicos do Mioceno-Plioceno, caso do arco Barroso (Mamani et al., 2010). As referidas estruturas são interpretadas como importantes paleocondutos hidrotermais na área do prospecto Chapi Chiara, observando-se também a existência de outros trends menos extensos, mas importantes condicionantes de núcleos de alteração argílica avançada (e.g., NE-SW, E-W e NNW-SSE - vide Figura 4A).

(4) Adicionalmente, alvos com características geológicas similares a Chapi Chiara aparecem nas adjacências deste prospecto, como é o caso do prospecto Cerro Millo (Hennig et al., 2008), e do alvo San Antonio de Esquilache. A disposição espacial da ocorrência dos paleoestratovulcões onde se localizam estes três alvos segue um trend NE-SW, como verificado na Figura 5A. Composicionalmente, Cerro Millo é caracterizado por zonas de alteração argílica avançada (quartzo, alunita, caulinita), argílica (esmectita, caulinita, quartzo) e propilítica (clorita, calcita, epidoto, quartzo), enquanto San Antonio de Esquilache caracteriza-se pela presença de zonas de alteração argílica avançada (alunita, caulinita) e argilica (ilita, esmectita) em associação com intrusão diorítica exposta, e marcada por potencial para $\mathrm{Cu}-\mathrm{Ag}-\mathrm{Au}$ e outros metais (cf., considerações em Carrino et al., 2015a).

(5) No modelo exploratório de Corbett e Leach (1998), a mineralogia hipógena principal da zona argílica avançada acima referida, além da argílica (domínio de ilita) e propilítica (domínio de clorita, carbonato, epidoto) tende a se formar em condições de maiores temperaturas num sistema epitermal de alta sulfetação, podendo ainda refletir uma proximidade, em profundidade, do limite de transição de um domínio epitermal para um mesotermal. Este contexto é consistente com a possível existência de intrusão(ões) diorítica(s) próxima(s) à superfície do prospecto Chapi Chiara, similarmente ao que já é observado no alvo San Antonio de Esquilache, com a atual exposição de intrusões na superfície deste paleoestratovulcão contemporâneo. Adicionalmente, esta associação com um possível sistema pórfiro em profundidade parece seguir o modelo geral proposto por Sillitoe (2010), que está mostrado na Figura 5B. 


\section{Considerações}

O exemplo de investigação geológica do prospecto Chapi Chiara por meio do emprego de dados multifonte revela o papel de destaque atrelado a técnicas como a espectroscopia de refletância e a susceptibilidade magnética, que aparecem como ferramentas de baixo custo, rápidas e complementares para o estudo de mineralogia hidrotermal.

Aliada a estas técnicas, e objetivando-se a espacialização de respostas mineralógicas e geológicas de detalhe, dados imageadores hiperespectrais e dados de malhas adensadas de magnetometria terrestre reforçam o papel central de dados desta natureza ao estudo prospectivo de alvos minerais, incluindo-se a determinação de mineralogia hipógena exposta em superfície, a definição de núcleos de alteração, e condicionantes estruturais que caracterizam a região de enfoque, além de possíveis alvos similares e potenciais adjacentes.

\section{Agradecimentos}

Agradecemos à FAPESP pela bolsa de doutorado da primeira autora (processo no. 2011/00106-8), e à Gold Fields Inc., especialmente ao geólogo Chico Azevedo, e às geólogas Teresa Guevara e Shirley Custódio, pela cessão de dados de sensoriamento remoto, de magnetometria terrestre, e acesso à área do prospecto.

\section{Referências}

Acosta, J., Quispe, J., Santisteban, A., Acosta, H., 2008. Épocas metalogenéticas y tipos de yacimientos metálicos en la margen occidental del sur del Perú: latitudes $14^{\circ} \mathrm{S}-$ $18^{\circ} \mathrm{S}$. Resúmenes 14th Congreso Peruano de Geología, Lima, Peru, 1-6.

Benavides-Cáceres, V., 1999. Orogenic evolution of the Peruvian Andes: The Andean cycle. In: Skinner, B.J. (Ed.), Geology of ore deposits of the Central Andes. Society of Economic Geologists, Boulder, 61-107.

Boardman, J.W., Kruse, F.A., Green, R.O., 1995. Mapping target signatures via partial unmixing of AVIRIS data. Proceedings $5^{\text {th }}$. Annual JPL Airborne Earth Science Workshop, Washington, USA, 23-26.

Carrino, T.A., Crósta, A.P., Toledo, C.L.B., Silva, A.M., Silva, J.L., 2015a. Geology and hydrothermal alteration of the Chapi Chiara prospect and nearby targets, southern Peru, using ASTER data and reflectance spectroscopy. Economic Geology, 110: 73-90.

Carrino, T.A., Crósta, A.P., Toledo, C.L.B., Silva, A.M., 2015b. Unveiling the hydrothermal mineralogy of the Chapi Chiara gold prospect, Peru, through reflectance spectroscopy, geochemical and petrographic data. Ore Geology Reviews, 64: 299-315.

Carrino, T.A., Crósta, A.P., Toledo, C.L.B., Silva, A.M., 2016 (submetido). Hydrothermal alteration of the Chapi
Chiara epithermal system, Peru: imaging spectroscopy and magnetic data. Economic Geology.

Corbett, G., Leach, T., 1998. Southwest Pacific rim goldcopper systems: structure, alteration and mineralization. Special Publication number 6. Society of Economic Geologists Inc., Bookcrafters, Chelsea.

Hedenquist, J.W., Arribas, A., Gonzalez-Urien, E., 2000. Exploration for epithermal gold deposits. Soc. Econ. Geol. Rev., 13: 245-277.

Hennig, D., Lehmann, B., Burgess, R., Tak, M.A.N., 2008. Geology, geochemistry and ${ }^{40} \mathrm{Ar} /{ }^{39} \mathrm{Ar}$ ages of the Cerro Millo epithermal high-sulfidation gold prospect, southern Peru. Ore Geology Reviews, 34: 304-316.

Loayza, D., Barreda, J., Crósta, A., Hedenquist, J., Morche, W., 2004. Late Miocene high sulfidation epithermal gold deposits of the Aruntani district, southern Peru. In: Muhling, J. (Ed.), SEG 2004 - Predictive Mineral Discovery Under Cover. University of Western Australia and Centre for Global Metallogeny Publication, Perth, Australia, 212-215.

Mamani, M., Wörner, G., Sempere, T., 2010, Geochemical variations in igneous rocks of the Central Andean orocline $\left(13^{\circ} \mathrm{S}\right.$ to $\left.18^{\circ} \mathrm{S}\right)$ : tracing crustal thickening and magma generation through time and space. Geological Society of America Bulletin, 122: 162-182.

Mundt, J.T., Streutker, D.R., Glenn, N.F., 2007. Partial unmixing of hyperspectral imagery: theory and methods. Proceedings. American Society of Photogrammetry AND Remote Sensing Annual Conference, Tampa, USA, 1-12.

Nabighian, N.M., 1972. The analytic signal of twodimensional magnetic bodies with polygonal cross section: its properties and use for automated anomaly interpretation. Geophysics, 37: 507-517.

Nabighian, N.M., 1974. Additional comments on the analytic signal of two-dimensional magnetic bodies with polygonal cross section. Geophysics, 39: 85-92.

Palácios, O.M., 1995, Estratigrafía. In: Palácios, O.M., Sánchez, A.F., Herrera, F.R. (Eds.), Geología del Perú, Boletín $n^{\circ} \quad 55$, Série A. Firmat S.A.-Editores \& Impresores, Lima, 45-86.

Santos, A., Baumgartner, R., Gaibor, A., Dusci, M., Azevedo, F., Gradim, R., Dunkley, P., Den Boer, D., Valer, R., 2011. Geology and mineralisation of the Au-CuAg Canahuire epithermal deposit, Chucapaca Project, southern Peru. Proceedings $11^{\text {th }}$. Society for Geology Applied to Mineral Deposits Biennial Meeting, Antofagasta, Chile, 1-4.

Sillitoe, R. H., 2010. Porphyry copper systems. Economic Geology, 105: 3-41.

White, N.C., Hedenquist, J.W., 1995. Epithermal gold deposits: styles, characteristics and exploration. Soc. Econ. Geol. Newsl., 23: 9-13. 


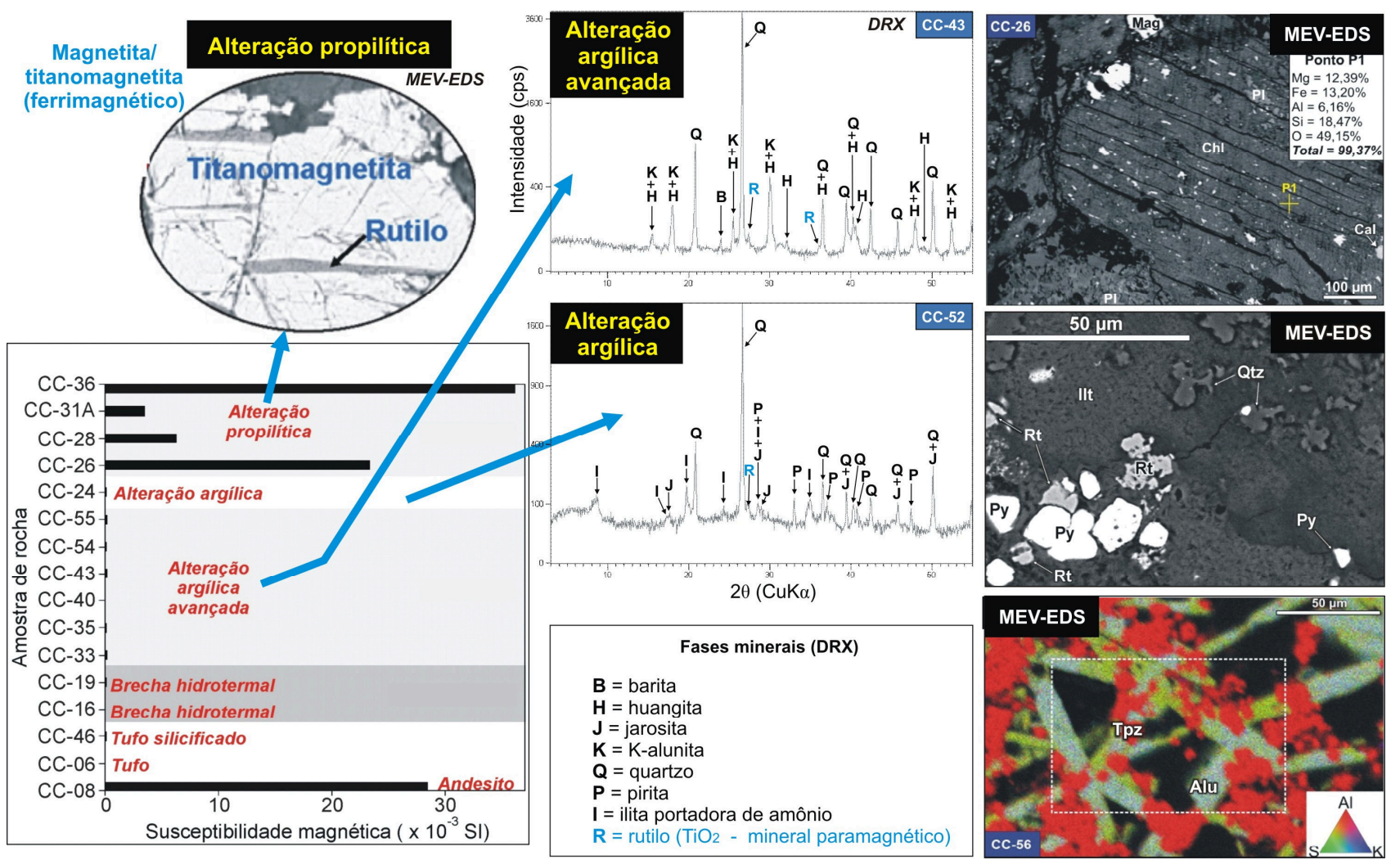

Figura 2. Síntese da caracterização de rochas alteradas na região do prospecto Chapi Chiara, mediante análises mineralógicas por MEV-EDS, difratometria de raios-X (DRX) e medidas de susceptibilidade magnética. Alu $=$ alunita, $\mathrm{Chl}=$ clorita, Ilt = ilita, Mag = magnetita, $\mathrm{PI}=$ plagioclásio, $\mathrm{Py}=$ pirita, $\mathrm{Qtz}=$ quartzo, $\mathrm{Rt}=$ rutilo, $\mathrm{Tpz}=$ topázio.
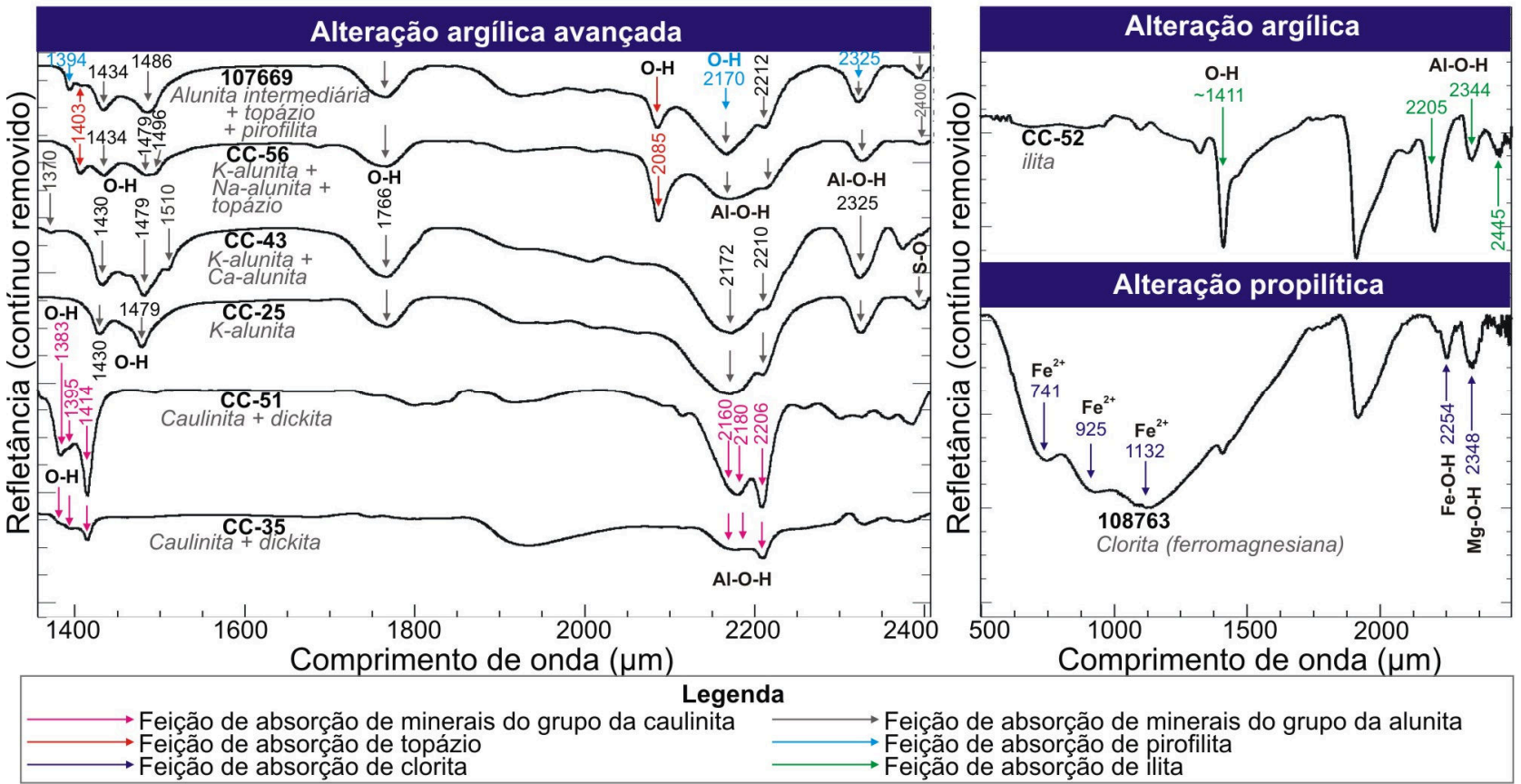

Figura 3. Exemplos de curvas de refletância de amostras de rochas alteradas em Chapi Chiara. Setas indicam as principais feições de absorção que discriminam as fases minerais indicadas. Discussão mais aprofundada acerca da análise sistemática de curvas espectrais coletadas na área do prospecto pode ser vista em Carrino et al. (2015b). 


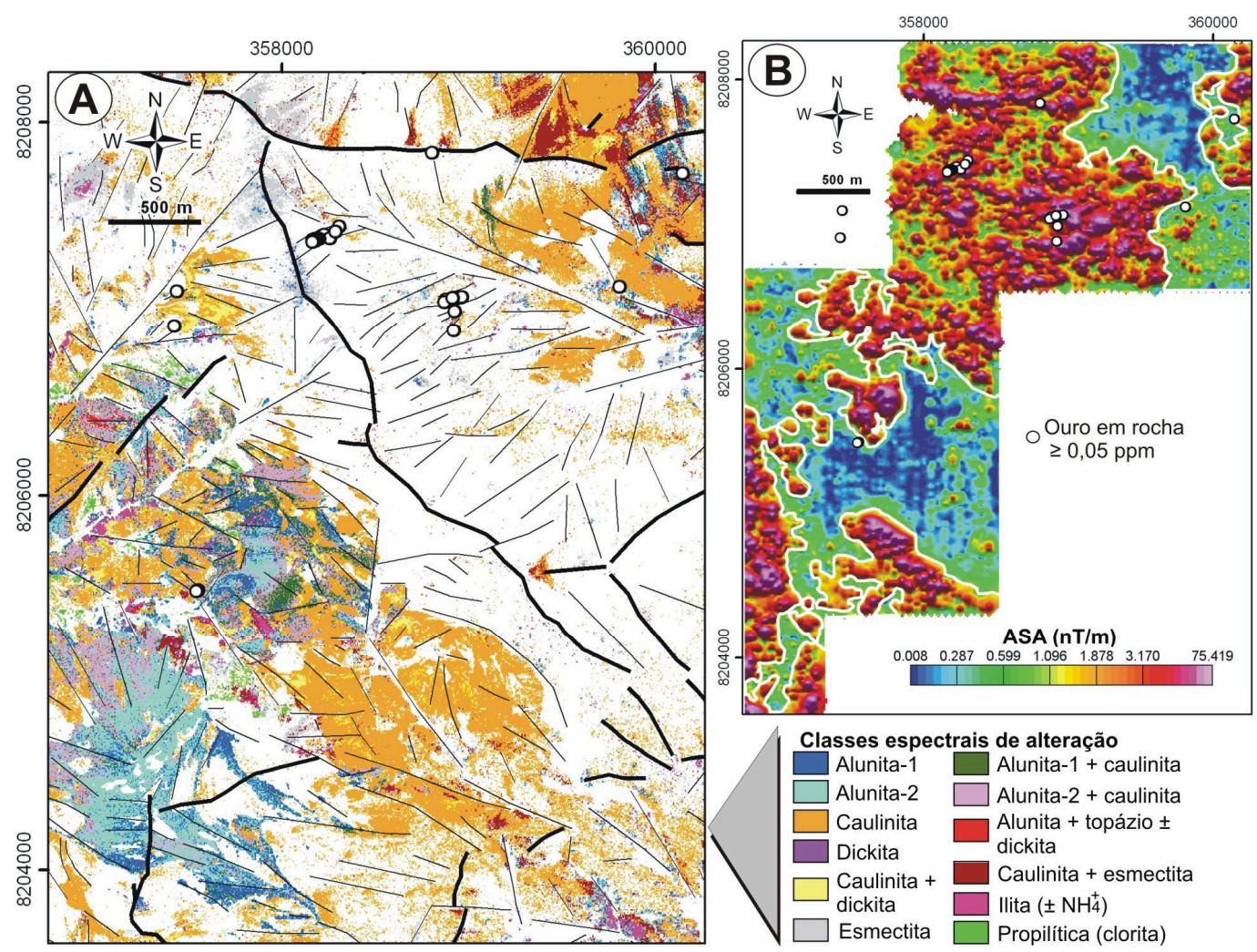

Figura 4. (A) Mapa de alteração hidrotermal do prospecto Chapi Chiara (simplificado de Carrino et al., 2016 - submetido), obtido a partir do processamento de imagens do sensor HyMap. (B) Mapa da amplitude do sinal analítico (ASA), com indicação de ocorrências de ouro e zonas desmagnetizadas/alteradas.
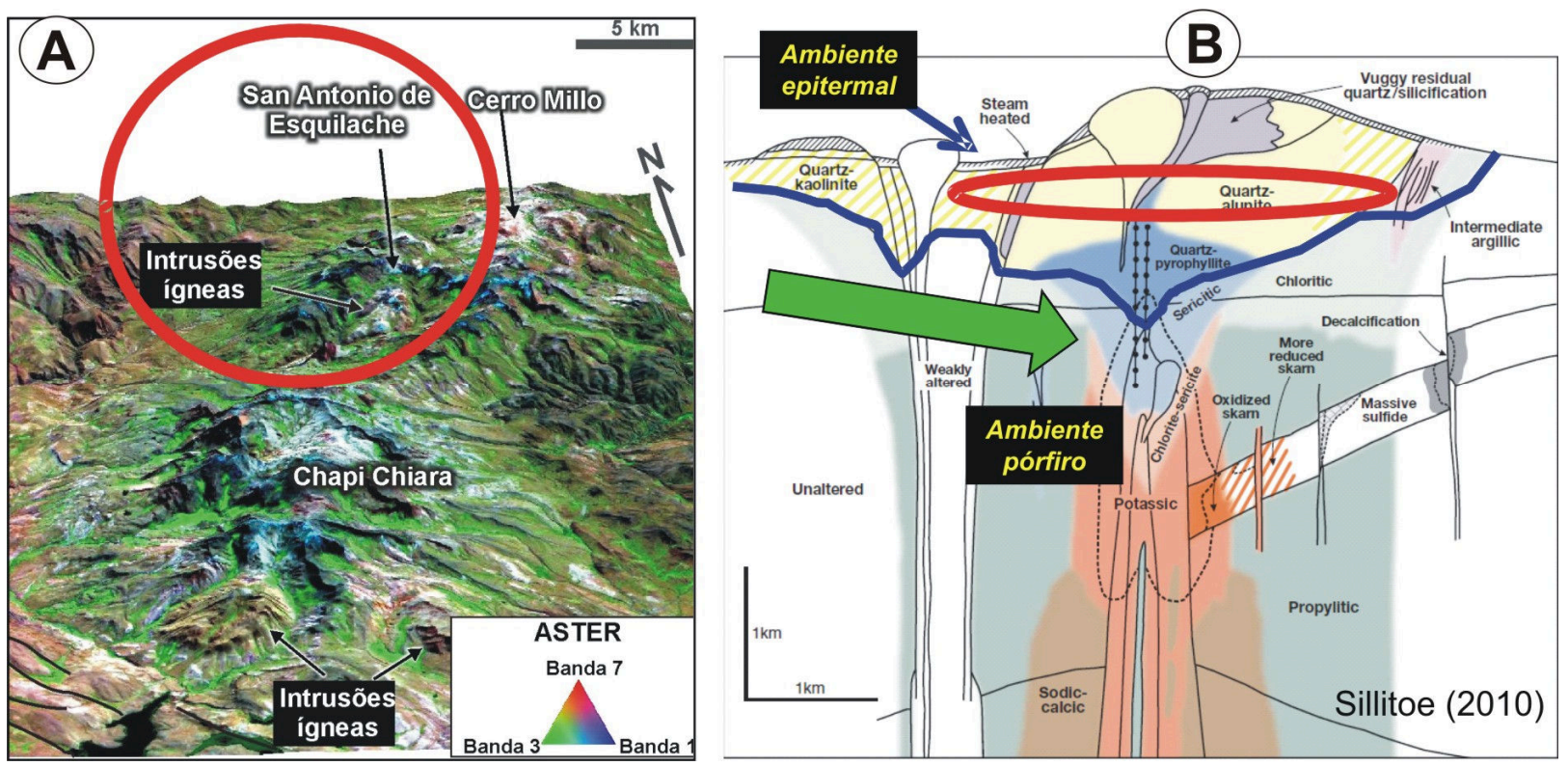

Figura 5. (A) Caracterização regional de paleoestratovulcões em imagem multiespectral do sensor ASTER (Advanced Spaceborne Thermal and Reflection Radiometer), onde se localizam os alvos Chapi Chiara, Cerro Millo, e San Antonio de Esquilache, incluindo-se a indicação da exposição em superfície de intrusão de diorito neste último (modificado de Carrino et al., 2015a). (B) Modelo de sistema epitermal-pórfiro (Sillitoe, 2010), e indicação de níveis de erosão estimados em Chapi Chiara (elipse em vermelho). 